\title{
Corela
}

Cognition, représentation, langage

HS-8 | 2010

L'interpellation

\section{L'Interpellation : genre de discours et statut illocutoire}

Caroline Mellet

\section{OpenEdition}

1 Journals

Édition électronique

URL : http://journals.openedition.org/corela/1719

DOI : 10.4000/corela. 1719

ISSN : $1638-573 \mathrm{X}$

Éditeur

Cercle linguistique du Centre et de I'Ouest - CerLICO

Référence électronique

Caroline Mellet, «L'Interpellation : genre de discours et statut illocutoire », Corela [En ligne], HS-8 |

2010, mis en ligne le 01 octobre 2010, consulté le 08 janvier 2020. URL : http://

journals.openedition.org/corela/1719; DOI : 10.4000/corela.1719

Ce document a été généré automatiquement le 8 janvier 2020.

\section{(c) (i) (2)(2)}

Corela - cognition, représentation, langage est mis à disposition selon les termes de la licence Creative Commons Attribution - Pas d'Utilisation Commerciale - Partage dans les Mêmes Conditions 4.0 International. 


\title{
L'Interpellation : genre de discours et statut illocutoire
}

\author{
Caroline Mellet
}

1 La communication que je propose s'inscrit dans le cadre d'une recherche plus vaste concernant les genres de discours parlementaires. Je me réfère à la réflexion du cercle de Bakhtine $^{1}$ associant très étroitement la diversité des activités humaines à la production de sortes de textes présentant des caractéristiques communes suffisamment importantes pour être regroupés en famille. Mon étude porte plus particulièrement sur la question de l'historicité des genres de discours, directement liée à l'évolution même des pratiques sociales. Cette caractéristique des genres a été maintes fois relevée : Bronckart (1996 : 76) note par exemple «le caractère fondamentalement historique (et adaptatif) des productions textuelles: certains genres tendent à disparaître (le récit épique), mais peuvent parfois réapparaître sous des formes partiellement différentes; certains genres se modifient ...; de nouveaux genres apparaissent (cf. le tract publicitaire) ; bref, les genres sont en perpétuel mouvement $»$.

2 C'est en quelque sorte ce mouvement que j'étudie au sein d'une activité sociale fortement institutionnalisée : l'activité parlementaire. On relève en effet une adaptation des formes génériques à l'évolution des contextes historiques et plus particulièrement politiques. C'est dans ce cadre que j'étudie l'interpellation. En tant que genre de discours réglé par l'institution parlementaire, l'interpellation a disparu, mais son étude représente à mes yeux un double intérêt :

3 - d'une part, il appartient à l'ensemble des genres de discours produits par l'institution politique française lorsqu'il s'agit de mettre différents pouvoirs en situation d'interlocution directe ${ }^{2}$. Cet ensemble comprend, par exemple, l'Adresse, octroyée par la Charte de $1814^{3}$ aux Chambres, pour suggérer ou affirmer leur opinion, ou encore, sous la cinquième République, les Questions au Gouvernement ${ }^{4}$, posées directement par les députés aux ministres présents dans l'hémicycle. Dans tous les cas, par l'institution d'un genre de discours dont le nom correspond à un acte de langage, l'institution politique entend régler et réguler l'interaction entre les pouvoirs et exprimer le type de rapports qu'elle entend instaurer. De ce point de vue, il est intéressant de comparer les Questions 
au Gouvernement ${ }^{5}$ et les interpellations pour comprendre l'évolution du rapport de forces entre le pouvoir exécutif et le pouvoir législatif. Les Questions au Gouvernement manifestent, par le choix même de l'acte de langage octroyé par les institutions politiques de la cinquième République, un moindre pouvoir que les interpellations, comme pratiques discursives sous les troisième et quatrième Républiques. En effet, la question est généralement décrite comme pourvue d'un statut illocutoire ambigu ${ }^{6}$ : d'un côté, elle constitue un acte agressif puisqu'il s'agit d'une incursion dans les réserves d'autrui. D'un autre côté, la question revient à manifester son ignorance, à faire l'aveu d'un manque que l'on demande à l'autre de combler. Le questionneur est ainsi amené à s'effacer pour susciter la parole de l'autre et lui laisser toute la place. L'interpellation concentre sans conteste une intensité illocutoire beaucoup plus forte. On attribue souvent le pouvoir de l'interpellation (comme genre de discours) à sa composante perlocutoire: en effet, lorsque l'interpellation est discutée au fond ${ }^{7}$, elle se termine par un ordre du jour, c'est-àdire un vote de confiance au Gouvernement. L'interpellation peut ainsi provoquer la chute du Gouvernement ${ }^{8}$. Il me semble néanmoins que les caractéristiques proprement illocutoires de l'interpellation doivent être prises en compte et donc décrites. Cela permettra de comprendre pourquoi le choix de cet acte de langage contribue à instaurer un rapport de domination du pouvoir législatif sur le pouvoir exécutif.

4 - d'autre part, la comparaison entre le genre politique de l'interpellation et des Questions au Gouvernement met en évidence des points communs, mais également des points de rupture qui permettent d'étudier les processus d'évolution des genres de discours au sein d'une institution. Bronckart souligne que les genres constituent « une sorte de réservoir de modèles textuels, auquel tout agent d'une action langagière devra nécessairement puiser » (1996:104). Son propos vaut pour l'appropriation par les locuteurs en un état synchronique donné, mais il reste valable dans une perspective diachronique. En l'occurrence, il permet de comprendre comment l'interpellation peut représenter l'un des modèles pour la constitution du nouveau genre qui apparaît sous la cinquième République : les Questions au Gouvernement.

5 Ce double intérêt que présente l'interpellation comme genre régulant des rapports de forces politiques et comme dispositif textuel disponible dans l'interdiscours pour la formation du genre des Questions au Gouvernement, m'amène à une interrogation plus précise sur le statut illocutoire de l'interpellation.

6 Je m'appuie ici sur un corpus limité, correspondant aux interpellations de la première session parlementaire ordinaire de l'année 1932. De ce fait, mes conclusions ne peuvent qu'être partielles. Elles demanderont à être étayées par une étude portant sur des interpellations à d'autres périodes. Ajoutons que le corpus est constitué des compte rendus des débats publiés au Journal officiel. Il s'agit donc d'un genre de discours particulier, comportant ses propres normes. Ce ne sont pas les interactions telles qu'elles se sont réellement déroulées que j'analyse, mais la représentation qui en est donnée par ce média.

7 Précisons rapidement les caractéristiques contextuelles nécessaires à la compréhension des exemples choisis: La France est gouvernée depuis 1928 par une majorité de droite. Plusieurs Présidents du Conseil se sont succédé. Au début de l'année 1932, c'est Laval qui est Président du Conseil ${ }^{9}$. Dans un contexte de crise économique mondiale, la France est préoccupée par la question des réparations ${ }^{10}$, que l'Allemagne refuse de payer. Une grande partie des interpellations de la session de 1932 porte sur la situation internationale et sur cette question. 
8 Je conduirai mon analyse en deux mouvements : je proposerai d'abord une première approche du statut illocutoire de l'interpellation par l'étude des conditions nécessaires à sa réalisation. Je considère néanmoins que la valeur illocutoire des actes de langage ne se stabilise et ne se négocie qu'en discours. C'est pourquoi, je poursuivrai, dans une deuxième partie, par l'étude de certaines réalisations discursives de l'interpellation comme genre. Cette étude permettra de préciser et d'affiner le statut illocutoire de l'acte d'interpeller.

\section{Le statut illocutoire de l'interpellation : conditions essentielles, conditions préliminaires et intensité illocutoire}

9 Reprenant certains aspects de la description que Searle (1972) propose des actes de langage indépendamment de tout contexte énonciatif, je propose d'étudier certaines conditions nécessaires à la réalisation de l'acte d'interpellation. Je m'intéresse principalement à trois aspects : les conditions essentielles, les conditions préliminaires et l'intensité illocutoire de l'acte.

Les conditions essentielles ${ }^{11}$ sont les suivantes :

11 interpeller revient pour L (le locuteur) à exprimer le désir que A (l'allocutaire) prenne en compte $\mathrm{L}$ dans son champ perceptif. Mais ce n'est pas tout. C'est un acte qui contient une deuxième condition essentielle :

12 - interpeller revient pour $\mathrm{L}$ à exprimer le désir que $\mathrm{A}$ interrompe son action en cours. J'emploie volontairement ce terme dans un sens très général qui englobe l'action physique, mais également l'action mentale, perceptive ou langagière ;

13 - ajoutons, enfin, que la réalisation de ce désir n'est pas forcément favorable à $\mathrm{A}$.

14 En ce qui concerne les conditions préliminaires ${ }^{12}$, elles peuvent être décrites de la façon suivante :

15 - A n'a pas pris encore en compte la présence de L dans son champ perceptif au moment de l'interpellation (d'une certaine manière, A est absent à L) ;

16 - A est déjà engagé dans une action ;

17 - L est à même d'interpeller (c'est-à-dire qu'il a l'autorité suffisante pour le faire).

18 Ajoutons, pour terminer, que l'intensité illocutoire ${ }^{13}$ est forte.

19 Cette description succincte de l'acte m'amène à une analyse en deux points :

20 Elle permet tout d'abord de catégoriser cet acte et, à mon sens, de le rapprocher de deux types d'actes de langage: en effet, l'interpellation peut être d'abord intégrée dans la classe des directifs. De manière plus précise, l'interpellation serait à classer dans la catégorie des demandes de faire. Pour Searle, la condition essentielle de la demande de faire revient à "essayer d'amener A à effectuer $\mathrm{C}$ ». "Demander, prier, supplier, réclamer, ordonner, commander, exiger ...revient à exprimer le souhait, ou le désir que $\mathrm{C}$ soit exécuté " (1972: 107). Cela correspond à la deuxième condition essentielle de l'interpellation. Précisons d'ailleurs que son intensité illocutoire (forte) rapproche davantage l'interpellation de l'acte d'ordonner que de celui de supplier, d'autant que le rapport d'autorité entre $\mathrm{L}$ et $\mathrm{A}$ est, semble-t-il, plutôt favorable à L. L'interpellation est donc à ranger parmi les actes agressifs, intrusifs, menaçants pour la face négative de $\mathrm{A}$. 
D'un autre côté, l'interpellation a des points communs avec l'acte de salutation. En effet, l'interpellation, comme la salutation, peut être assimilée à l'expression d'une reconnaissance en même temps qu'un désir d'être reconnu. Néanmoins, l'interpellation n'implique pas, comme la salutation, un rapport de réciprocité : d'une part, parce que l'interaction que l'interpellation initie n'est pas forcément souhaitée par A. Elle constitue un coup de force de la part de L. Et surtout, L n'attend pas d'autre réponse de la part de A que l'interruption de son action ou de son propre mouvement. Notons encore que, contrairement à la question, qui implique que le locuteur cède la parole et la place à l'allocutaire, l'interpellation ne sollicite l'autre que pour le détourner de lui-même.

Une telle description de l'interpellation pourrait l'assimiler à une simple interruption. Il semble pourtant que les deux actes ne se confondent pas : l'interruption ne comporte pas forcément la condition essentielle 1 , à savoir le désir de prise en compte de $\mathrm{L}$ dans le champ perceptif de A. Des échanges entre étudiants, par exemple, peuvent interrompre un autre échange, sans qu'il y ait désir de reconnaissance, sans qu'il y ait interpellation. L'interruption n'est pas forcément adressée; il me semble que l'interpellation l'est toujours.

23 La description de l'interpellation comme acte de langage permet la catégorisation de l'acte mais elle rend également possible de préciser le pôle de l'interaction privilégié par l'acte d'interpellation. Il constitue certes une énonciation adressée (il s'agit donc bien d'un acte allocutif), mais dans la mesure où il revient à tenter d'interrompre l'autre dans son expansion, dans son désir d'accomplissement de soi au profit de l'interpellateur, il manifeste davantage le désir de rapporter l'attention sur le pôle élocutif. Il semble d'ailleurs que cette caractéristique de l'interpellation la différencie de l'adresse qui peut être considérée comme un simple appel à l'autre, sans précision du pôle privilégié.

\section{Analyse discursive de l'interpellation}

Je propose à présent une étude discursive à travers l'étude de l'interpellation comme genre de discours. Cette étude passe d'abord par l'analyse succincte des conditions de création de ce genre discursif. Pour cela, il est souhaitable de faire la distinction entre deux significations du terme interpellation au sein de l'institution parlementaire :

l'interpellation peut s'entendre tout d'abord comme acte d'interpeller, au cours d'une prise de parole publique dans l'hémicycle. Cet acte peut se réaliser par l'usage du performatif «j'interpelle» ou par d'autres moyens (l'apostrophe, l'utilisation de la deuxième personne, etc) ;

L'interpellation peut également désigner un genre de discours: il s'agit sans doute d'abord d'une pratique discursive stabilisée, liée à des contraintes spécifiques et engendrant des productions textuelles similaires ; puis d'un genre de discours institué, et recevant comme tel une nomination.

Si l'utilisation du verbe interpeller comme performatif ( «j'interpelle») est attestée dans les comptes rendus de débats à l'époque révolutionnaire, l'interpellation, comme genre de discours est d'une création plus tardive. Au début de l'année 1831, sous la monarchie de Juillet, le député Mauguin affirme (par une interpellation -au sens de l'acte-) le droit des députés à interpeller le Gouvernement. En autorisant la discussion, la Chambre consacre implicitement ce droit, et commence à réglementer, par l'usage, les normes discursives et interactionnelles de l'interpellation (cette fois-ci en tant que genre) : le 
sujet et le jour de l'interpellation doivent être annoncées à l'avance par exemple. Si les différents régimes usant de l'interpellation vont contribuer progressivement à la réglementation de ce genre de discours (l'Assemblée législative de 1848 fixe ainsi que les interpellations doivent être rédigées par écrit et remises au Président), c'est surtout la pratique qui en façonne les caractéristiques. Il n'est pas possible, dans le cadre de ce présent article, de faire une étude historique de la pratique du genre de l'interpellation sous les différents régimes politiques. Je voudrais simplement relever deux points :

1/ L'interpellation est attribuée au Parlement. Elle lui donne ainsi l'initiative de l'interaction avec le pouvoir exécutif. Celui-ci, quelle que soit sa nature (républicaine ou monarchique), n'est pas, de manière essentielle, en situation de demande, ni même de dialogue, mais d'action. L'interpellation constitue ainsi l'un des moyens politiques permettant la limitation du pouvoir exécutif. Elle est bien perçue comme l'une des armes empêchant l'expansion de l'action politique ; elle est avant tout un acte d'interruption.

2/ L'interpellation est toujours perçue comme l'expression d'un pouvoir important accordé au pouvoir législatif. Lorsque le pouvoir exécutif devient dominant, l'interpellation est supprimée (c'est le cas, par exemple, en 1852, pendant la phase la plus autoritaire du second Empire), ou remplacée par un genre de discours moins agressif et intrusif (comme les Questions au Gouvernement sous le régime présidentiel de la cinquième République).

Ces caractéristiques historiques de l'interpellation mettent en évidence certains aspects de l'acte d'interpeller (la condition essentielle d'interruption, le degré élevé de force illocutoire), mais elle doit être complétée par une étude discursive.

31 L'analyse du corpus met en évidence une grande variété des actes de discours réalisés : variété entre les différentes prises de parole, variété à l'intérieur des interventions mêmes, chaque interpellation intégrant un certain nombre d'actes. Néanmoins, la variété des actes composant l'interpellation peut être, en partie seulement, résorbée par leur intégration dans un acte directeur ${ }^{14}$, c'est-à-dire un acte central qui donne à l'ensemble de l'intervention sa valeur pragmatique globale. Nous posons ici la nécessité de recourir à la notion de macro-acte de discours pour l'interprétation d'unités pragmatiques de dimension supérieure à la phrase. L'utilisation d'une telle notion ${ }^{15}$ pose un certain nombre de difficultés. La plupart des critères retenus pour déterminer la valeur pragmatique globale d'une intervention ne sont pas entièrement satisfaisants : le critère interactionnel (c'est l'enchainement qui permet de définir cette valeur) comporte une part d'incertitude, car il peut amener à confondre l'enchaînement effectué avec l'appréciation qu'en ferait le chercheur. La présence d'un préfixe performatif explicitant l'intention du locuteur est un indice intéressant, mais il doit être également soumis à l'analyse: ce qui se présente directement comme une demande d'information ${ }^{16}$, par exemple, peut servir à réaliser indirectement un autre macro-acte. Enfin, il est certain qu'un discours peut cumuler plusieurs valeurs illocutoires. Néanmoins, cette notion m'apparaît utile pour l'appréhension de la valeur pragmatique de l'interpellation. Malgré la variété des interventions, quelques régularités apparaissent, et je voudrais attirer plus particulièrement l'attention sur quatre caractéristiques se dégageant de l'analyse du corpus :

32 -Tout d'abord, il apparaît que l'interpellation n'est pas favorable à A : certes, les interpellations peuvent contenir des actes favorables à l'allocutaire (le corpus contient en particulier un remerciement et un éloge ponctuel ${ }^{17}$ au chef du gouvernement), mais il 
s'agit d'occurrences rares et généralement remarquées, considérées comme moins attendues. Louis Marin, dans son interpellation du 21 janvier 1932, le souligne d'ailleurs :

«Monsieur le Président du conseil, vous devez penser, après avoir entendu les interpellations d'aujourd'hui, qu'il est extrêmement difficile de vous interpeller :... les interpellateurs vous comblent de compliments.»

L'analyse du corpus confirme que l'interpellation politique est plus agonale qu'irénique ; elle manifeste un désaccord et exprime le souhait d'une entrave à la pleine réalisation de l'action de l'allocutaire.

34 - L'interpellation est une prise de parole longue. La durée d'un genre de discours doit être considérée comme un indicateur important. Bien sûr, la notion de durée est relative (aux normes culturelles et individuelles, et à l'objet -en l'occurrence un discours d'assemblée-). Néanmoins, dans le cadre de l'analyse des interactions en général et des interactions politiques en particulier, elle peut être un indicateur de la relation taxémique entre les interlocuteurs. De plus, elle est un indice du pôle de l'interlocution privilégié. De ce point de vue, une comparaison peut être faite entre le genre des Questions au Gouvernement sous la cinquième République et les interpellations dans notre corpus. Les Questions au Gouvernement sont contraintes par une extrême brièveté (2mns et 30s). D'autre part, la dénomination du genre de discours (la "question») impose que le locuteur cède, à un moment donné, la parole à l'allocutaire, qu'il lui permette "d'occuper le terrain »( Kerbrat-Orecchioni 1991: 29 ), déplaçant ainsi l'objet de l'interaction vers le pôle de l'allocutaire. Au contraire, les interpellations ne connaissent pas de limitation de temps. De manière significative, le Président des débats octroie la parole à l'orateur par la phrase suivante : «La parole est à M. X pour développer son interpellation ». L'interpellation est conçue comme un développement, une expansion temporelle de soi, qui n'attend d'autre réponse que la reconnaissance et l'attention. L'interpellation ne suppose pas de manière impérative que l'on cède la parole à l'allocutaire. C'est bien le pôle du locuteur qui est favorisé.

- L'interpellation est un discours adressé. Les normes parlementaires prescrivent en effet l'allocutaire. Eugène Pierre, considéré comme l'un des meilleurs connaisseurs de l'institution parlementaire, rappelle en 1924, dans son Traité de droit politique, électoral et parlementaire, que les « interpellations de collègue à collègue sont interdites ». Dans notre corpus, plusieurs interventions du Président ont pour objet la bonne observation de cette règle. Il en est ainsi le 21 janvier 1932, par exemple, lors de l'interpellation du député Jean Autrand:

«M. le président. Vous interpellez nos collègues et non le Gouvernement. Vos observations sont sans rapport avec le débat. "

Dans la mesure où les normes parlementaires prescrivent l'allocutaire, celui-ci n'a pas besoin d'être identifié au moyen de l'apostrophe. Nous pouvons constater cependant que la plupart des interventions sont rythmées par de nombreuses apostrophes. A titre d'exemple, nous pouvons nous intéresser plus particulièrement à l'interpellation de Léon Blum le 19 janvier 1932: il s'agit d'une longue prise de parole qui a pour fonction de démontrer la nécessité de lier la question des réparations à celle de la dette envers les Etats-Unis. Sa proposition est d'accepter que l'Allemagne suspende ce qu'elle doit à la France, à la condition que les Etats-Unis suspendent ou retardent le paiement de la dette contractée par la France. Dans son discours, la fonction essentielle des apostrophes n'est pas, sauf exception, la ratification de l'allocutaire, ni même son identification. Il est d'ailleurs intéressant de noter que, sur les 23 apostrophes que contient son discours, 16 
sont constituées de l'apostrophe «messieurs», qui n'a pas, dans le contexte parlementaire de l'époque, de référent stable : elle peut référer aussi bien aux membres du Gouvernement, qu'aux députés, et elle englobe parfois le président de l'assemblée luimême. Cette apostrophe contient les unités sémiques minimales nécessaires à l'identification du discours comme adressé.

La fonction d'implication est plus importante. Cela est particulièrement net dans les passages du discours de Léon Blum, où celui-ci accompagne l'apostrophe d'une injonction destinée à prendre en compte la situation de l'Allemagne. L'apostrophe a alors pour fonction de proposer une nouvelle orientation du regard afin de contempler d'une nouvelle manière le référent (c'est-à-dire ici l'Allemagne) :

« représentez-vous, messieurs, qu'il est peut-être difficile à un pays plongé dans le degré de désordre, de misère et de désespoir où est aujourd'hui descendue l'Allemagne ... Représentez-vous qu'il est difficile à ce peuple d'imaginer que sa situation puisse changer un jour au point que l'ère de ses paiements à l'étranger puisse recommencer. »

Il s'agit bien ici d'une implication, qui va, au-delà de la seule fonction d'appel, jusqu'au guidage de l'attention vers une autre perception : tandis que de nombreux parlementaires décrivent l'Allemagne comme un pays toujours dangereux pour la France, en plein réarmement, Léon Blum propose un autre éclairage, en insistant au contraire sur l'impuissance et la misère de l'Allemagne. Cet exemple montre bien que l'interpellation est un discours adressé, mais elle a avant tout pour fonction une réorientation de l'attention. Il s'agit bien ici d'interrompre une action (perceptive) pour en proposer une autre.

Dans certains cas, la réorientation de l'attention se charge non pas d'éclairer l'objet d'une nouvelle manière, mais de ramener le regard de l'allocutaire vers le locuteur. Les passages les plus polémiques de l'interpellation de Léon Blum usent d'apostrophes dont la fonction est d'impliquer l'allocutaire dans l'acceptation d'une conclusion opposée à celle qu'il est supposé défendre. En évoquant la possibilité de renoncer aux réparations (et donc de faire preuve de "solidarité et d'humanité » à l'égard de l'ennemi d'hier), Léon Blum a suscité de nombreuses protestations. Même s'il présente également l'autre partie de l'alternative (affirmer une grande rigueur), l'ensemble de son propos est perçu comme scandaleux. Le passage qui suit constitue une réplique à ses opposants :

«Et voilà, messieurs, l'alternative, que je n'avais pas tort, je crois, de qualifier de tragique et dont, pas plus que d'autres, mais, au moins, autant qu'aucun autre, nous pouvons, nous, sentir le poids" (source ? ?:il s'agit toujours de la même interpellation de L. Blum)

40 La forme négative " je n'avais pas tort » constitue une négation polémique, s'opposant à l'affirmation contraire que Léon Blum déduit des mouvements de protestations lorsqu'il évoque la possibilité de prendre en compte le point de vue de l'Allemagne. L'apostrophe qui l'accompagne a alors une nette fonction argumentative puisqu'il s'agit d'impliquer les allocutaires dans une conclusion favorable au locuteur, et de les contraindre à accepter l'assertion (l'alternative est tragique, selon le député socialiste, car elle conduit de toute manière à favoriser la propagande hitlérienne). Encore une fois, il s'agit d'un mouvement qui les invite à abandonner leur position pour adopter celle de Léon Blum.

41 - Enfin, l'interpellation peut souvent être interprétée comme un macro-acte d'injonction. L'intervention de Léon Blum contient un certain nombre d'actes qui doivent être 
interprétés en fonction de l'acte directeur qui les intègre. Celui-ci peut se dégager à partir du plan de texte, qui répond à une organisation rigoureuse en trois parties :

1/ la première est un constat : l'Allemagne ne veut plus payer les réparations à la France ;

$43 \quad 2$ /dans la deuxième partie, les solutions antagonistes généralement avancées (exiger coûte que coûte le paiement ou faire preuve d'humanité et annuler la dette) sont renvoyées dos à dos, rejetées comme nuisibles. L'évocation des deux possibilités s'accompagne de l'accomplissement d'un acte de menace (dans les deux cas, il s'agit du risque de favoriser la propagande hitlérienne);

3/ enfin, la dernière phase de l'argumentation consiste en un macro-acte directif que l'on peut classer dans la catégorie des injonctions. Cette partie se décompose en une première injonction, ayant valeur de critique : le Gouvernement doit changer de politique. Suit une seconde injonction qui constitue une proposition : lier la question des réparations à celle de la dette envers les Etats-Unis. Enfin, cette injonction est renforcée par un acte de menace :

« vous perdrez les élections si vous ne changez pas de politique».

L'affirmation de la valeur illocutoire de question énoncée en début de discours, les questions qui closent l'intervention («Qu'allez-vous choisir?») ne font que masquer une intervention plus intrusive et plus contraignante. L'injonction constitue bien l'expression d'un souhait pressant de l'interruption de l'action en cours. Elle trouve ici son vrai sens politique, tout en éclairant, de manière essentielle, la valeur principale de l'acte d'interpeller.

\section{Conclusion}

L'étude de l'interpellation comme genre de discours historiquement situé rend compte de sa fonction politique principale: portée par le pouvoir législatif, elle est une arme empêchant l'expansion de l'action inhérente au pouvoir exécutif. Ce rôle politique du genre de l'interpellation permet de mettre en relief la valeur illocutoire de l'acte dont il porte le nom: la valeur fondamentale de l'acte d'interpeller est celle d'une interruption, d'une limitation de l'expansion de l'autre au profit du locuteur.

La relation qui est établie ici entre acte de langage et genre de discours pourra être approfondie: ces deux notions, parfois confondues, entretiennent des liens qui demandent à être éclaircis, notamment pour tous les genres de discours, qui, comme c'est le cas ici, portent un nom qui est aussi celui d'un acte de langage. Une analyse fine des occurrences du performatif «j'interpelle» (ou "nous interpellons») pourrait, par exemple, mettre en évidence les moments où s'opère la transformation de l'usage du verbe comme marque possible de la réalisation du genre de discours (et non plus seulement comme simple performatif). Cette étude permettrait, en outre, de contribuer à saisir le processus d'émergence d'un nouveau genre de discours au sein d'une sphère d'activité. 


\section{BIBLIOGRAPHIE}

Austin J. L. (2002), Quand dire, c'est faire, Paris, Le Seuil (Première édition 1962, How to do things with words, Oxford).

Bakhtine M. (Volochinov) (1977), Le Marxisme et la philosophie du langage, Paris, Minuit.

Benveniste E., (1977), Problèmes de linguistique générale (T.2), Paris : Gallimard.

Bota C., Bronckart J.-P. (2007), Volochinov et Bakhtine : deux approches radicalement opposées des genres de textes et de leur statut, Linx 56, Nanterre.

Branca-Rosoff S. (1999), Types, modes et genres : entre langue et discours, Langage et société 89, 5-24.

Bronckart J.-P. (1996), Activité langagière, textes et discours, Lausanne : Delachaux et Niestlé.

Cadoux C. (1982), Droit constitutionnel et institutions politiques : les régimes politiques contemporains, Paris : Cujas.

Détrie C., (2006), De la non-personne à la personne : l'apostrophe nominale, Paris : CNRS Editions.

Facq-Mellet C. (2005), Analyse discursive des Questions au Gouvernement, thèse de doctorat (direction J.-F. Jeandillou), Paris-X Nanterre (en cours d'édition).

Kerbrat-Orecchioni C. (dir.), (1991), La Question, Lyon : PUL.

Kerbrat-Orecchioni C. (2001), Les Actes de langage dans le discours : théorie et fonctionnement, Paris : Nathan.

Moirand S. (2003), Quelles catégories descriptives pour la mise au jour des genres de discours, site du GRIC, Université Lyon 2-Lumière et ENS LSH, [http://gric.univ-lyon2.fr/Equipe1/actes/ journee_genre.htm].

Muselier F (1956), Regards neufs sur le Parlement, Paris : Le Seuil.

Nef F. (1980), Notes pour une pragmatique textuelle, Communications 32, 183-189.

Pierre E. (1924), Traité de droit politique, électoral et parlementaire, Paris : Loysel.

Récanati F. (1981), Les Enoncés performatifs, Paris : Minuit.

Searle J.R. (1972), Les Actes de langage, Paris : Hermann (Première édition Speech acts, Cambridge, CUP, 1969).

\section{NOTES}

1. Je m'en tiens ici au nom mis traditionnellement en avant pour la paternité des réflexions concernant les genres de discours. Pour une remise en cause de cette conception traditionnelle, lire le dernier numéro de la revue Linx 56, Linguistique des genres. Le Programme de Bakhtine et ses perspectives contemporaines, et en particulier l'article plutôt convaincant de Bota et Bronckart (73-90).

2. Je ne prends en compte que les genres relevant de l'histoire contemporaine, à partir de la Révolution française. L'étude pourrait néanmoins être étendue avec intérêt à des genres en 
pratique lors de périodes plus anciennes, comme par exemple les remontrances lors des Etats Généraux à l'époque moderne (voir à ce sujet Daubresse S., 2005, Le Parlement de Paris ou la voix de la raison, Genève, Droz, 2005).

3. Sous le règne de Louis XVIII (Cadoux 1982).

4. voir à ce sujet Facq-Mellet $C$., Analyse discursive des questions au Gouvernement, thèse de doctorat soutenue publiquement en novembre 2005 (en cours de publication).

5. Les Questions au Gouvernement (ou Questions d'actualité) ont été instituées en 1974 par Valery Giscard d'Estaing. Pour plus de détails sur la création et la caractérisation de ce genre de discours politique, voir Neidhart et al. (1997).

6. «Si l'on se situe au niveau de la question-en-général, on ne peut que reconnaître son 'ambivalence taxémique'» (Kerbrat-Orecchioni, 1991, 28).

7. Une interpellation peut parfois être seulement « inscrite à l'ordre du jour pour fixation de date, ce qui signifie qu'on ne la discute pas au fond: on discute seulement du jour où on la discutera » (Muselier 1956, 95-96).

8. «L'adoption d'un ordre du jour défavorable au gouvernement signifiait refus de la confiance et obligation en fait pour le Gouvernement de démissionner » (Cadoux 1982, 310).

9. Il vient de former un nouveau gouvernement, sans A. Briand, considéré par beaucoup comme «le ministre de la paix».

10. c'est-à-dire, en vertu de l'application du traité de Versailles, l'argent que l'Allemagne doit verser à la France en compensations des dommages sur le sol français pendant la première guerre mondiale.

11. Ils s'agit de conditions qui, pour Searle (1972), font partie de la définition même de l'acte de langage.

12. Il s'agit des conditions dont dépend la réussite de l'acte : «...la condition préliminaire nous apprend (au moins en partie ce qu'il (l'acte) sous-entend en accomplissant cet acte. De façon générale, le locuteur sous-entend, par l'accomplissement d'un acte illocutionnaire quelconque, que les conditions préliminaires de l'acte sont satisfaites. » (Searle 1972, 110)

13. Entendue comme degré d'intensité de l'acte.

14. On appelle ainsi l'acte central «qui donne à l'ensemble de l'intervention sa valeur pragmatique globale ». (Kerbrat-Orecchioni $2001: 60$ )

15. Voir à ce sujet Nef (1980).

16. Plusieurs interpellations débutent ainsi par une explicitation de l'intention pragmatique du locuteur: «Messieurs, mon interpellation aura un objet très strictement déterminé. Dans quelques jours s'ouvre la conférence de Lausanne. Dans peu de semaines s'ouvrira la conférence du désarmement, à Genève. Je suis à la tribune pour demander au Gouvernement, au nom de mes amis, quelle sera sa position dans ces deux conférences." (Léon Blum, Journal officiel, compte rendu des débats de la séance du 19 janvier 1932, p. 46)

17. «Et qui donc aujourd'hui peut aller à travers le monde redresser les énergies chancelantes, porter la parole d'espoir, rendre la foi au lendemain? Le chef de ce Gouvernement, qui, sous le signe de notre politique, inspire partout le respect de la France.» (Jean Autrand, Journal officiel, compte rendu des débats de la séance du 21 janvier 1932, p. 83) 


\section{RÉSUMÉS}

L'article présenté ici propose d'aborder la question de la valeur pragmatique de l'interpellation à travers l'étude du genre de discours politique du même nom, en vigueur, dans les institutions françaises, jusqu'à la fin de la quatrième République. L'analyse se déroule en deux temps. On se propose d'abord d'étudier l'acte d'interpeller, en passant en revue les conditions (essentielles et préliminaires) nécessaires à sa réalisation. L'étude de l'interpellation comme genre de discours (à partir de l'analyse du corpus limité aux interpellations de la première session parlementaire ordinaire de 1932) permet de confirmer le sens appréhendé : l'interpellation apparaît fondamentalement comme un acte d'interruption, de limitation de l'expansion de l'autre au profit du locuteur.

\section{INDEX}

Keywords : speech acts, essential conditions, genders of discourse, global speech act, preparatory conditions

Mots-clés : acte de langage, condition essentielle, condition préliminaire, genre de discours, condition essentielle macro-acte

\section{AUTEUR}

\section{CAROLINE MELLET}

Université Paris 10 